\title{
Effect of radiation and porosity parameter on hydromagnetic flow due to exponentially stretching sheet in a porous media
}

\author{
B. Mukherjee ${ }^{1}$, N. Prasad ${ }^{2 *}$ \\ ${ }^{1}$ Department of Applied Mathematics, Indian School of Mines, Dhanbad,, INDIA \\ ${ }^{2}$ Department of Applied Mathematics, Indian School of Mines, Dhanbad,, INDIA \\ *Corresponding Author: e-mail:narayan.ismmaths@gmail.comTel+919608138672
}

\begin{abstract}
The aim of this paper is to study the momentum and the heat transfer characteristics in incompressible electrically conducting boundary layer flow over an exponentially stretching sheet under the effect of magnetic field with thermal radiation through porous medium. The governing boundary layer equations are converted into self-similar nonlinear ordinary differential equations, using similarity transformations in exponential form and then solved numerically using shooting method. The velocity profile, skin friction-co-efficient and rate of heat transfer are computed numerically and then graphically studied with respect to similarity variable $(\eta)$ for different cases of velocity ratio parameter $(\beta)$
\end{abstract}

Keywords: Boundary layer, unsteady flow, exponentially stretching surface, porous medium, thermal radiation.

DOI: http://dx.doi.org/10.4314/ijest.v6i1.7

\section{Introduction}

Flow of incompressible viscous fluid and heat transfer phenomena over stretching sheets have received great attention due to the abundance of practical application in the chemical and manufacturing process like Aerodynamics, Extrusion of plastic sheets, Continuous Casting of metals, glass fibers and paper production. The study of Magneto hydrodynamics flow and Heat transfer of an electrically conducting fluid has attracted the interest of many researchers due to extensive industrial application. The study of hydrodynamic flow of an electrically conducting fluid caused by the deformation of the wall of a vessel containing a fluid is of considerable interest in a modern metallurgical and metal-working process.

Crane (1970) was the first to consider the boundary layer flow caused by a stretching sheet which moves with a velocity varying linearly with the distance from a fixed point. The heat transfer aspect of this problem was investigated by Carragher and Crane (1982), under the conditions when the temperature difference between the surface and the ambient fluid is proportional to a power of the distance from a fixed point. Numerous studies such as Pavlov (1977), Gupta et al. (1977), Chen et al. (1988), Pop et al.(1996), Vajrvelu (2001), Cortell (2007), Zhang et al.(2011) and Bhatacharyya (2011) have been conducted later to extend the pioneering work of Crane (1970). Last few decades in almost all investigations on the flow over a stretching sheet, the flow occurs because of linear variation of stretching velocity of the flat sheet. So the boundary layer flow induced by an exponentially stretching sheet is not studied much through it is very important and realistic flow frequently appeared in many engineering process. Magyari and Keller (1999) first considered the boundary layer flow due to an exponential stretching sheet and they also studied the heat transfer in the flow taking exponentially varied wall temperature. Elbashbeshy (2001) numerically examined the flow and heat transfer over an exponentially stretching surface considering wall mass suction. Khan and Sanjayanand (2005) studied the flow of viscoelastic fluid sheet with viscous dissipation effect. Partha et al. (2005) obtained a similarity solution for mixed convection flow past an exponentially stretching surface by taking into account the influence of viscous dissipation on the convective transport. Sanjayanand and Khan (2006) discussed the effects of heat and mass transfer on the boundary layer flow of viscoelastic fluid. Al-Odat et al. (2006) explained the effect magnetic field on thermal boundary layer on an exponentially 
stretching continuous surface with an exponential temperature distribution. Recently, Sajid and Hayat (2008) showed the influence of thermal radiation on the boundary layer flow past an exponentially stretching sheet and they reported a series solution for velocity and temperature using HAM.

Hiemenz (1911) first studied the steady flow in the neighbourhood of a stagnation-point. Chiam. (1994)considered a problem which is a combination of the works of Hiemenz (1911) and Crane (1970), i.e. the stagnation-point flow towards a stretching sheet taking identical stretching rate of the sheet and strain rate of the stagnation-point flow and he found no boundary layer structure near the sheet. Mahapatra and Gupta(2001) reinvestigated stagnation-point flow towards a stretching surface. They reported in their research work that a boundary layer is formed when stretching velocity is less than the free stream velocity. As the stretching velocity exceeds the free stream velocity than an inverted boundary layer is formed. Mahapatra et al. (2002) studied heat transfer in stagnation-point flow on stretching sheet with viscous dissipation effect. Viscous incompressible fluid striking on a permeable stretching surface with heat generation or absorption has been studied by Attia (2000).the same stagnation-point flow towards a stretching sheet with different stretching and straining rates and found two kinds of boundary layer near the sheet depending on the ratio of the stretching and straining rates.

Many researchers have analyzed magnetic field parameter on two-dimensional stagnation point flow of a viscous incompressible stagnation point flow of a viscous incompressible fluid. Attia (2007) investigated effect of increasing magnetic field on velocity boundary. Effects of Ohmic heating and viscous dissipation on steady flow with variable free stream has been investigated by Singh et al.(2009). Many other phenomenon for effects of heat transfer flows have been discussed in detail by Bejan et al. (2006), and White (2006). Free convection heat transfer with radiation effect near the isothermal stretching sheet and over a flat sheet near the stagnation point have been investigated, respectively, by Ghaly et al. (2002) and Pop et al. (2004). They found that a boundary layer thickness increases with radiation. The radiative effect on the heat transfer from an arbitrarily stretching surface with nonuniform surface temperature in a porous medium has been studied by Rashad(2007) .

Motivated by the above investigations and possible applications, it is of interest in the present work to study the problem of the effect of thermal radiation and heat transfer over an unsteady two dimensional viscous, incompressible flow of an electrically conducting fluid over an exponentially stretching surface embedded in a porous medium. The approach uses boundary layer theory along with the similarity procedure to find and compare feasible solution. The motion of the fluid is generated due to exponentially stretching of the sheet with the application of two equal \& opposite forces which are applied along the $\mathrm{x}$-axis so that the wall is stretched keeping the origin fixed. Fluid is considered in the influence of transverse magnetic field and thermal radiation. The linear stretching of the sheet is assumed because of its simplicity in the modeling of the flow and heat transfer over stretching surface which also permits the similarity solution and are useful in understanding the interaction of flow field with temperature field.

\section{Formulation of the Problem}

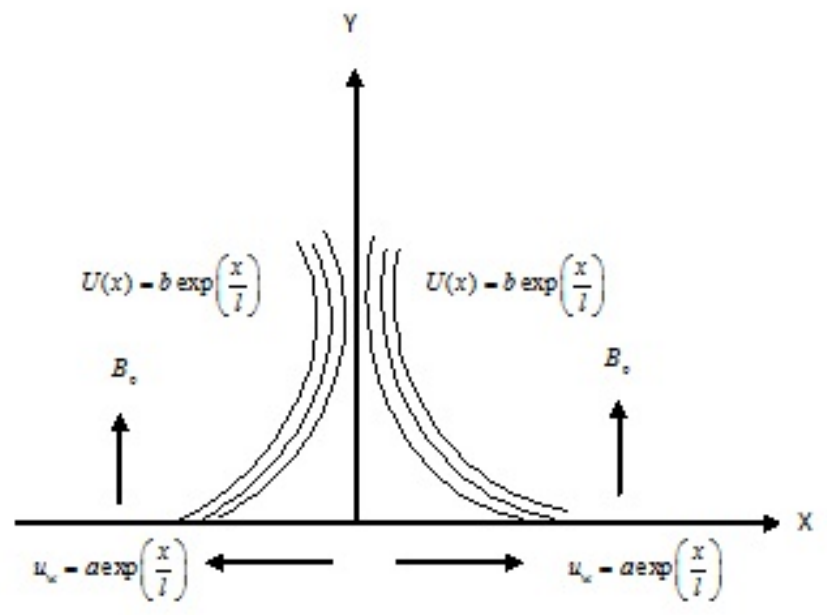

Fig. 1 Geometry of the problem

A steady two-dimensional flow of an incompressible viscous electrically conducting fluid over a continuous exponentially stretching sheet with velocity $U_{w}(x)$ in an exponential free stream with velocity $U(x)$, assuming that the plate has a surface temperature $T_{0}$ and is placed in a fluid of uniform ambient temperature $T_{\infty}$, has been considered as shown in Figure 1 .The sheet coincides with the plane $y=0$ and the flow confined to $y>0$. The motion of the fluid is generated due to exponentially stretching of the sheet with the application of two equal \& opposite forces which are applied along the $\mathrm{x}$-axis so that the wall is stretched 
keeping the origin fixed. Here, in the present analysis there is no lateral velocity or pressure gradient far from the stretching surface. we have taken $\mathrm{x}$-axis along the wall in the direction of motion of the flow, the y-axis being normal to it and $u$ and $v$ are tangential and normal velocity components respectively. A uniform magnetic field of strength $B_{0}$ is applied normally to the stretching surface which produces magnetic effect. It is also assumed that the fluid is weakly electrically conducting so that the induced magnetic field is negligible, which is justified for MHD flow at small magnetic Reynolds number. Under the usual boundary layer approximations, the flow and heat transfer with the radiation effects (Bansal, 1977; Schlichting et al.,1999.) are governed by the following equations:

$$
\begin{aligned}
\frac{\partial u}{\partial x}+\frac{\partial v}{\partial y} & =0 \\
u \frac{\partial u}{\partial x}+v \frac{\partial u}{\partial y} & =-\frac{1}{\rho} \frac{\partial p}{\partial x}+v \frac{\partial^{2} u}{\partial y^{2}}-\frac{\sigma B_{0}^{2}}{\rho} u-\frac{v}{\lambda} u \\
-\frac{1}{\rho} \frac{\partial p}{\partial y} & =0 \\
u \frac{\partial T}{\partial x}+v \frac{\partial T}{\partial y} & =\frac{k}{\rho c_{p}} \frac{\partial^{2} T}{\partial y^{2}}-\frac{1}{\rho c_{p}} \frac{\partial q_{r}}{\partial y}+\mu\left(\frac{\partial u}{\partial y}\right)^{2}
\end{aligned}
$$

Here, eq. (3) implies that pressure is independent of $y$, means pressure can be taken from the potential of flow i.e. in the free stream because pressure is independent of thickness of the boundary layer i.e. pressure is same at any point of the line perpendicular to the stretching sheet inside the boundary layer.

The stretching velocity $U_{w}(x)$ and free stream velocity $U(x)$ are respectively given by (Bhattacharyya and Moukhopadhyay. 2011)

$$
\begin{gathered}
u=U_{w}=a \exp \left(\frac{x}{l}\right), \\
u=U(x)=b \exp \left(\frac{x}{l}\right),
\end{gathered}
$$

Here, in the free stream $u=U(x)=b \exp \left(\frac{x}{l}\right)$, eq. (2) reduces to.

$$
U \frac{\partial U}{\partial x}=-\frac{1}{\rho} \frac{\partial p}{\partial x}-\frac{\sigma B_{0}^{2}}{\rho} U-\frac{v}{\lambda} U
$$

Eliminating $-\frac{1}{\rho} \frac{\partial p}{\partial x} \quad$ from eq. (2) and (5), we get,

$$
u \frac{\partial u}{\partial x}+v \frac{\partial v}{\partial y}=U \frac{\partial U}{\partial x}+v \frac{\partial^{2} u}{\partial y^{2}}-\frac{\sigma B_{0}^{2}}{\rho}(u-U)-\frac{v}{\lambda}(u-U)
$$

For the present problem, where the stretching of the boundary surface is assumed to be such that the flow directional velocity is of exponential order of the flow directional, we employ the following boundary conditions

$$
\begin{aligned}
& u=U_{w}=a \exp \left(\frac{x}{l}\right), \quad v=0, \quad T=T_{\infty}+T_{0} \exp \left(\frac{x}{2 l}\right) \quad \text { at } y=0 \\
& u=U(x)=b \exp \left(\frac{x}{l}\right), \quad T=T_{\infty} \quad \text { at } y=\infty
\end{aligned}
$$

Here, eq. (7) implies that stretching sheet velocity and temperature varies exponentially with a distance from a fixed point with no slip condition and eq. (8) implies that outside of the boundary layer the stretching sheet velocity attained the full stream velocity $U$ and temperature attained the uniform ambient temperature $T_{\infty}$.

where $\rho$ is the fluid density, $v$ is the kinematic viscosity, $\mu$ is the dynamic viscosity, $T$ is the temperature, $k$ is the thermal conductivity, $c_{p}$ is the specific heat and $q_{r}$ is the radiative heat flux. $T_{0}$ and $T_{\infty}$ are respectively the temperatures at and far away from the plate and $l$ is a constant. 
Employing the Rosseland approximation for radiation [Chen. et al (2008)] expressed as,

$$
q_{r}=-\frac{4 \sigma}{3 \alpha} \frac{\partial T^{4}}{\partial y}
$$

where $a$ is mean absorption co-efficient and $\sigma$ is the Stefen Boltzman constant. It is assumed that the temperature difference within the flow sufficiently small such that $T^{4}$ can be expressed as a linear function of temperature which after expanding using Taylor's series about $T_{\infty}$ and neglecting the higher order term, reduces to

$$
T^{4}=4 T_{\infty}^{3} T-3 T_{\infty}^{4}
$$

Hence the change in radiative flux with respect to $y$ has been obtained as,

$$
\frac{\partial q_{r}}{\partial y}=-\frac{16}{3 \alpha} \frac{T_{\infty}^{3} \sigma}{\rho c_{p}} \frac{\partial^{2} T}{\partial y^{2}}
$$

and equation (3) reduces to

$$
u \frac{\partial T}{\partial x}+v \frac{\partial T}{\partial y}=\frac{k}{\rho c_{p}} \frac{\partial^{2} T}{\partial y^{2}}+\frac{16}{3 \alpha} \frac{T_{\infty}^{3}}{\rho c_{p}} \frac{\partial^{2} T}{\partial y^{2}}+\mu\left(\frac{\partial u}{\partial y}\right)^{2}
$$

The equation of continuity is satisfied if a stream function $\psi(x, y)$ is chosen such that

$$
u=\frac{\partial \psi}{\partial y} \text { and } v=-\frac{\partial \psi}{\partial x}
$$

By introducing the following dimensionless co-ordinate variable (Khan and Sanjayanand. 2005) viz;

$$
\begin{gathered}
\eta=y \sqrt{\frac{a}{2 v l}} \exp \left(\frac{x}{2 l}\right) \\
\psi(x, y)=\sqrt{2 v l} \exp \left(\frac{x}{2 l}\right) f(\eta) \\
T=T_{\infty}+T_{0} \exp \left(\frac{x}{2 l}\right) \theta(\eta)
\end{gathered}
$$

where $\eta$ is the similarity variable, $\psi$ is the stream function, $\theta$ is the dimensionless temperature and $f$ is the dimensionless stream function. The above momentum and energy equations by using equations (13) to (15) are made free from dimension \& reduces to

$$
\begin{gathered}
f^{\prime \prime \prime}+f f^{\prime \prime}-2 f^{\prime 2}-2(M+N)(f-\beta)+2 \beta^{2}=0 \\
\left(1+\frac{4}{3 R}\right) \theta^{\prime \prime}+P_{r}\left[f \theta^{\prime}-f^{\prime} \theta+E f^{\prime 2}\right]=0
\end{gathered}
$$

with the boundary condition;

$$
\begin{array}{rlrl}
f=0, f^{\prime} & =1, \theta=1 & \text { at } \eta=0 \\
f^{\prime}=\beta, \theta=0 & \text { at } \eta=\infty
\end{array}
$$


where, dash represent differentiation with respect to $\eta$ and $\beta=\frac{b}{a}$ is the velocity parameter, $M=\frac{\sigma B_{0}^{2} l}{\rho u_{w}}$ is magnetic parameter, $N=\frac{v l}{u_{w} \lambda}$ is the porosity parameter, $R=\frac{k \alpha}{4 \alpha T_{\infty}^{3}}$ is the radiation parameter $P_{r}=\frac{\mu c_{p}}{k}$ is the Prandtl number, $E=\frac{\alpha^{2}}{T_{0} c_{\rho}}$ is the Eckert number.

The skin friction co-efficient $c_{f}$ and the Nusselt number $N u_{x}$ are two important physical quantity and are defined as

$$
c_{f}=\frac{2 \tau_{w}}{\left(u_{w}\right)^{2}}, N u_{x}=\frac{x q_{w}}{T_{w}-T_{\infty}}
$$

where the skin friction $\tau_{w}$ and heat transfer from the sheet $q_{w}$ are given by

$$
\tau_{w}=\mu\left(\frac{\partial u}{\partial x}\right)_{y=0}, q_{w}=-k\left(\frac{\partial T}{\partial y}\right)_{y=0}
$$

By using equation (21) and (20), the skin friction co-efficient $c_{f}$ and the Nusselt number $N_{u_{x}}$ reduces to

$$
\begin{gathered}
c_{f} \sqrt{R_{e_{x}}}=f^{\prime \prime}(0) \\
N u_{x} \frac{N}{\sqrt{R_{e_{x}}}}=-\theta^{\prime}(0)
\end{gathered}
$$

To assess the accuracy of the present method, comparison with previously reported data available in the literature has been made. It is clear from Table 1 that the numerical values of $-\theta^{\prime}(0)$ in the present paper for $\operatorname{Pr}=1$ when $M=0, N=0, R=0$ and $E=0$ are in good agreement with results obtained by Mahapatra et al. (2002). From Table 1 it is clear that the scheme used in this paper is stable and accurate.

\section{Numerical Simulation}

In order to solve the set of non linear differential equations (16) and (17) subjected to the boundary conditions (18) and (19), the most efficient numerical shooting technique with Runge Kutta scheme is been used where it is most important to choose the appropriate finite values of $\eta \rightarrow \infty$.The solution process is repeated with another large value of $\eta_{\infty}$ until two successive values of $f^{\prime \prime}(0)$ and $\theta^{\prime}(0)$ up to the desired significant value. The last value of $\eta_{\infty}$ is chosen as appropriate value of the limit $\eta \rightarrow \infty$ for that particular set of parameters. The differential equations (16) and (17) were first converted into a set of five first-order simultaneous equations. To solve this system five initial conditions are required but there are only three initial conditions, $f(0)$ and $f^{\prime}(0)$ on $f^{\prime}(\eta)$ and $\theta(0)$ on $\theta(\eta)$. Still there are two initial conditions $f^{\prime \prime}(0)$ and $\theta^{\prime}(0)$ are required, which are not prescribed. However the values $f^{\prime \prime}(\eta)$ and $\theta^{\prime}(\eta)$ are known at $\eta \rightarrow \infty$. Shooting technique has been employed to find the two unknown initial values utilizing these two ending boundary conditions. After finding the required boundary conditions, the problem has been solved numerically using Runge-Kutta scheme.

\section{Results and Discussion}

To analyse the results numerical computations have been carried out by using the method described in the previous section for various value of velocity parameter $\beta$, magnetic parameter $M$, porosity parameter $N$, Prandtl number $P_{r}$, Radiation parameter $R$ and for Eckert number $E$.

It is evident from Fig 2 (b), that when $\beta>1$ the flow has a boundary layer structure and the thickness of the boundary layer decreases with the increase in $\beta$. Moreover the straining motion near the stagnation motion for $\beta>1$, increase so the acceleration of the external stream increases which causes to decrease the thickness of the boundary layer with increase in $\beta$ and as a result the horizontal velocity increases with the increase of $\beta$. 
On the other hand, when $\beta<1$ the flow has an inverted boundary layer as shown in Fig 2(a). These results agree with those reported by Mahapatra and Gupta (2002). In this case, the stretching velocity of the sheet exceeds the velocity of the external stream. It is also observed that no boundary layer is formed when $\beta=1$.

The magnetic parameter $M$ represent the importance of magnetic field on the flow. The presence of transverse magnetic field sets in Lorentz force, which results in retarding force on the velocity field and there for as $M$ increases, so does the retarding force and hence velocity decreases as shown in Fig.3 (a), when $\beta<1$. When $\beta>1$ which just opposite of $\beta<1$, expected velocity increases with increase of magnetic parameter as shown in Fig.3 (b). This explain the results presented in Table.4 is that the shear stress at the sheet decreases due to increase in magnetic parameter when $\beta<1$, while it increases with the increase in magnetic parameter when $\beta>1$.

Fig. 4(a) and Fig. 4(b) represent the influence of the porous medium on horizontal velocity. In these figure the graphical representation of horizontal velocity for different value of the porosity parameter for two set of value $\beta<1$ and $\beta>1$ is obtained.

It is found that for $\beta<1$, the horizontal velocity $f^{\prime}(\eta)$ decreases with the increase of $N$ i.e. porosity parameter resistance to the flow since it restricts the motion of the fluid along the surface, with increasing the value of $N$, the thickness of the velocity boundary layer increase, so the velocity decreases with the increases of the value of $N$.This is due to the fact that the effect of porous medium which opposes the flow also increases and leads to enhance deceleration of the flow, but horizontal velocity increases with the increase of $N$ when $\beta>1$.

Fig. 5(a) and Fig. 5(b) represent the effect of Prandtl number $P_{r}$ on temperature profile for both cases $\beta<1$ and $\beta>1$ respectively. Prandtl number defines the ratio of momentum diffusivity to the thermal diffusivity. It is noticed that as $P_{r}$ increases, the temperature decreases for both cases. This is because, physically, if $P_{r}$ increases, the thermal diffusivity decreases and these phenomena lead to the decreasing of energy ability that reduced the thermal boundary layer thickness.

From Fig. 6(a) and Fig. 6(b) it is very clear that with increasing value of the radiation parameter $R$, the thermal boundary layer thickness decreases and the heat flux of the surface increases since the temperature profiles become steeper. The effect of radiation parameter for both cases $\beta<1$ and $\beta>1$, at a particular point is to reduce the temperature significantly in the flow region. The increase in radiation parameter is equivalent to the release of heat energy from the flow region and so the fluid temperature decreases as the thermal boundary layer thickness become thinner.

From Fig.7 (a) and Fig.7 (b) we observe that with increasing value of Eckert number $E$ temperature increases for both cases $\beta<1$ and $\beta>1$ respectively. Eckert number, physically, is a measure of frictional heat in the system. Hence the thermal regime with larger $E$ values is subjected to rather more frictional heating causing a source of rise in temperature.

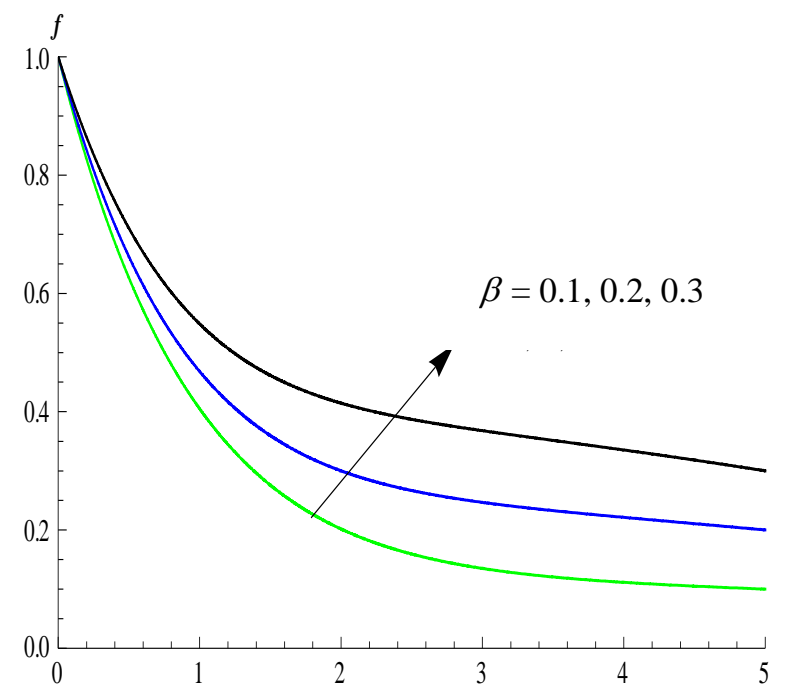

(a)

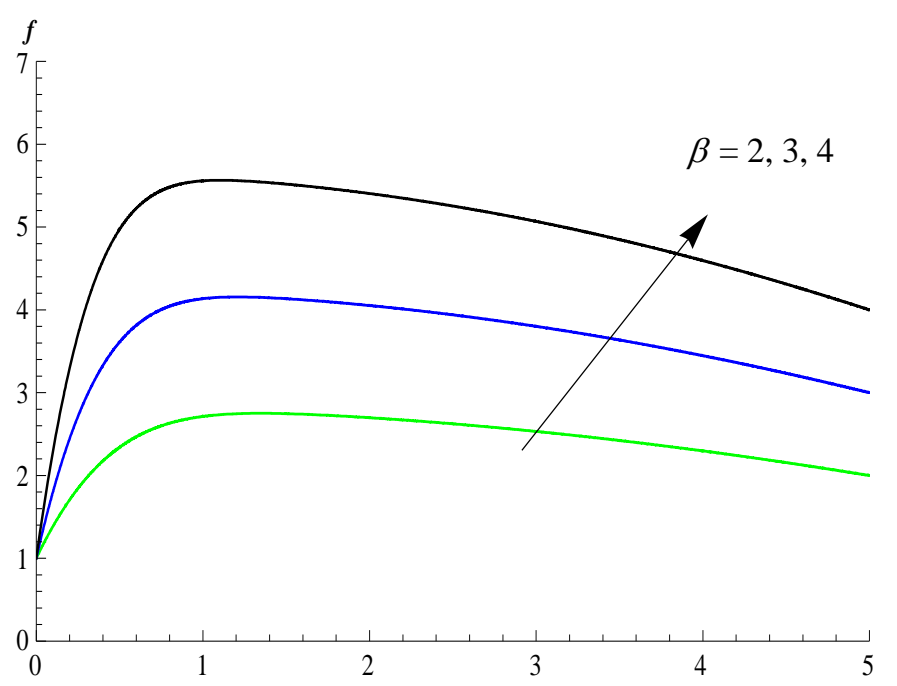

(b)

Fig. 2. Velocity profile $f^{\prime}(\eta)$ for different values of (a) $\beta<1$ and (b) $\beta>1$ for fixed value of $N=0, M=0, P_{r}=0, R=0$ and $E=0$. 


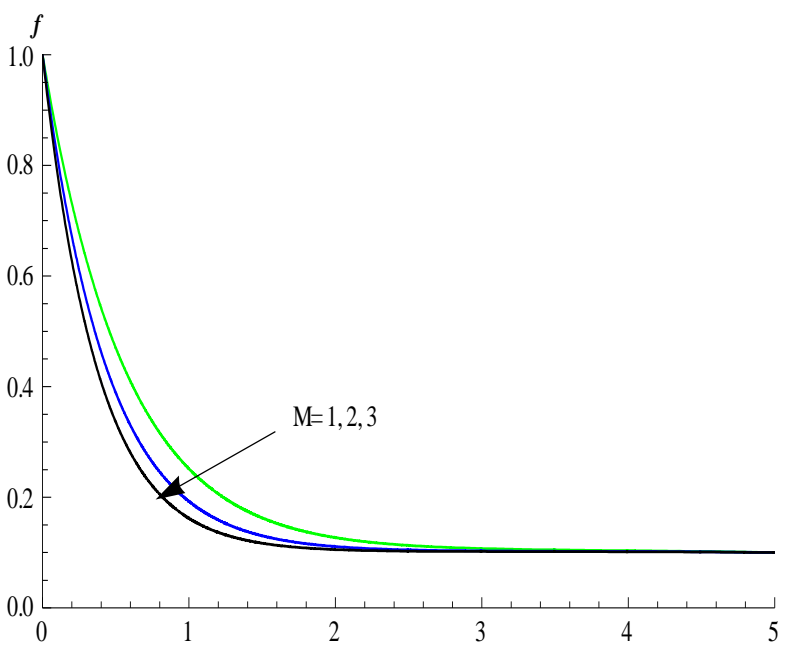

(a)

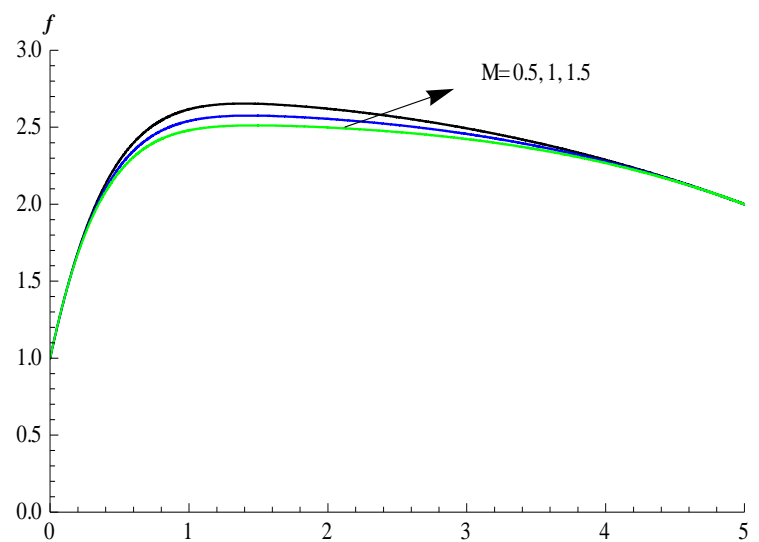

(b)

Fig. 3. Velocity profile $f^{\prime}(\eta)$ for different values of $M$ by taking (a) $\beta=0.1$ and (b) $\beta=2$ for fixed value of $N=1, P_{r}=1, R=1$ and $E=0.1$.

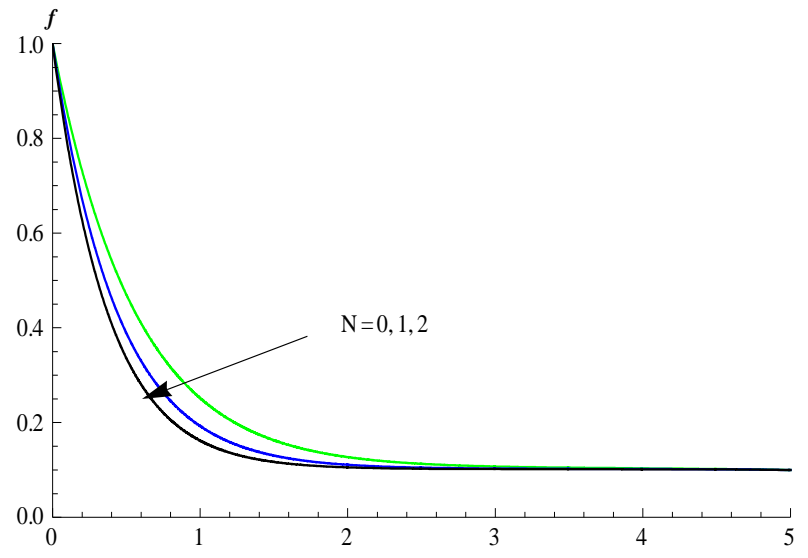

(a)

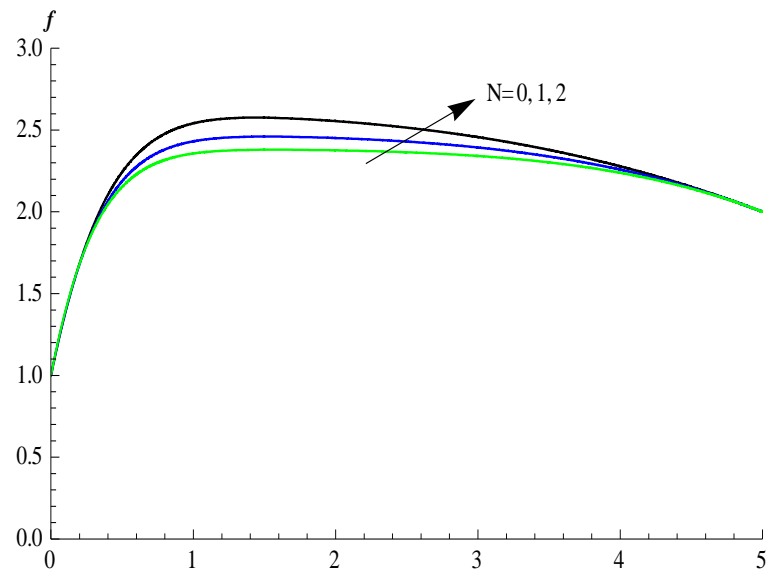

(b)

Fig.4. Velocity profile $f^{\prime}(\eta)$ for different values of $N$ by taking (a) $\beta=0.1$ and (b) $\beta=2$ for fixed value of $M=1, P_{r}=1, R=1$ and $E=0.1$

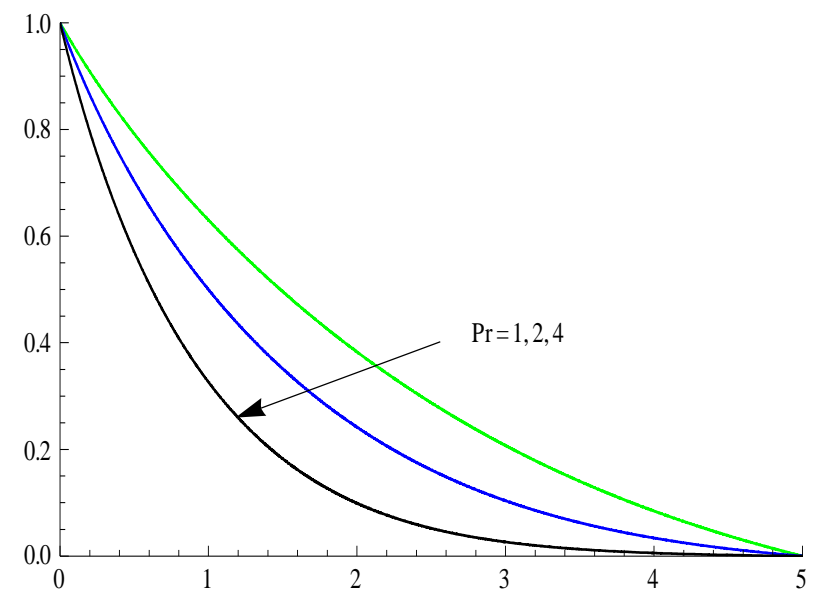

(a)

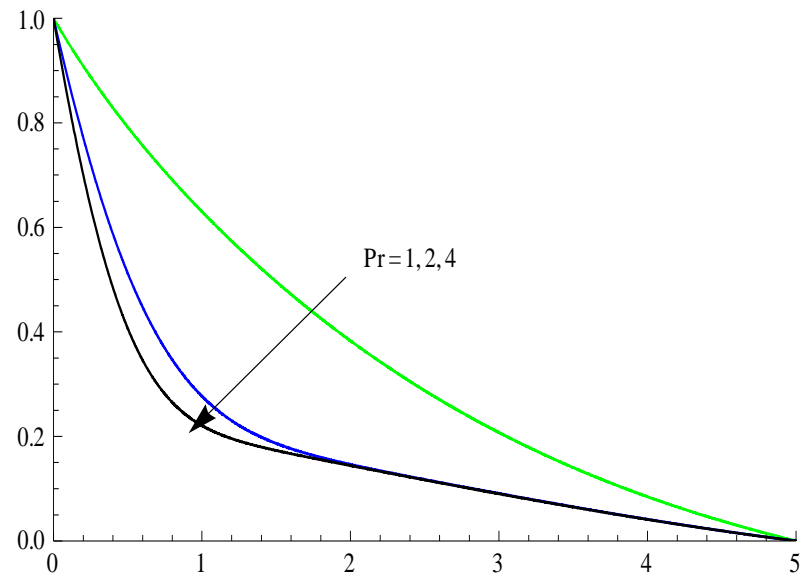

(b)

Fig.5. Temperature profile $\theta(\eta)$ for different values of $P_{r}$ by taking (a) $\beta=0.1$ and (b) $\beta=2$ for fixed value of $M=1, N=1, R=1$ and $E=0.1$ 


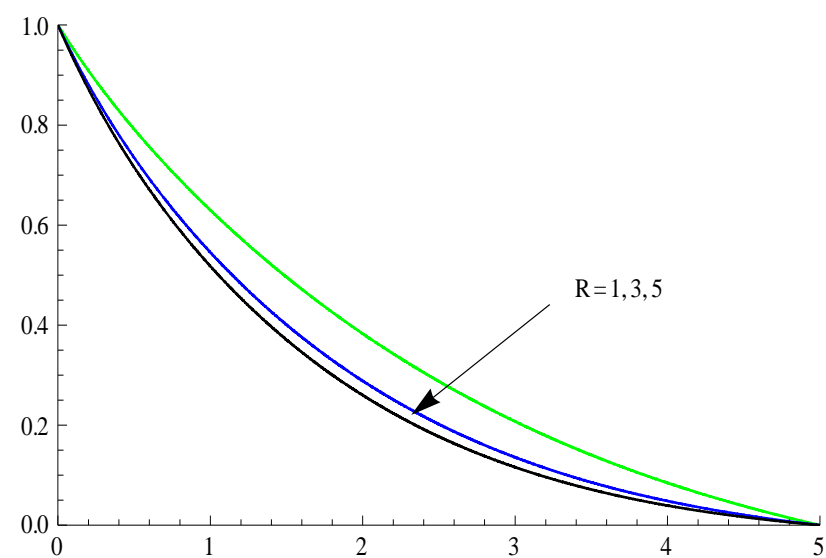

(a)

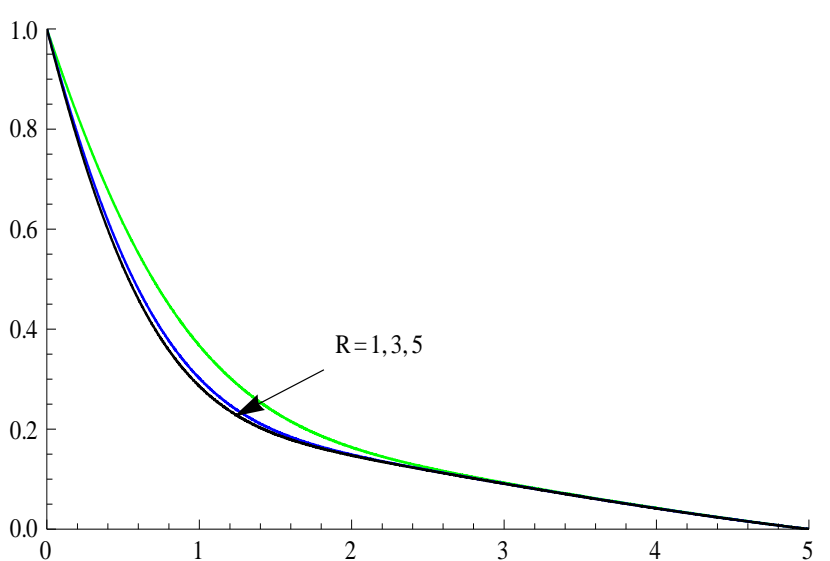

(b)

Fig.6. Temperature profile $\theta(\eta)$ for different values of $R$ by taking (a) $\beta=0.1$ and (b) $\beta=2$ for fixed value of $M=1, N=1, P_{r}=1$ and $E=0.1$

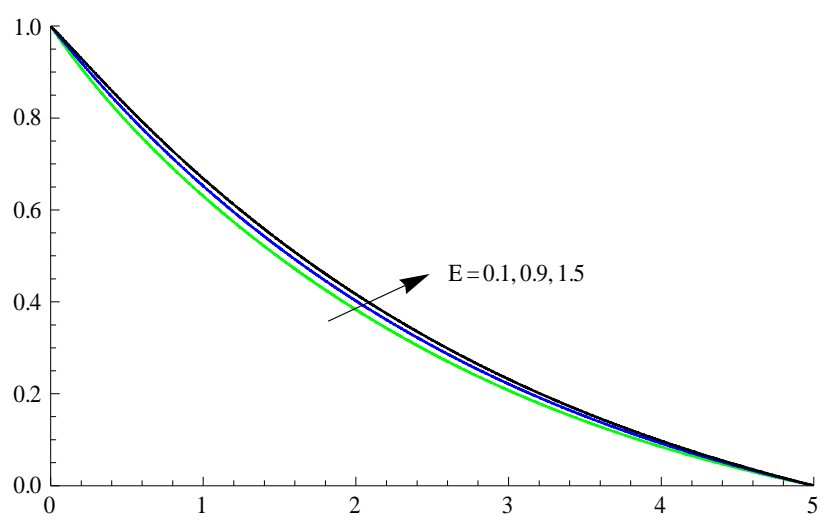

(a)

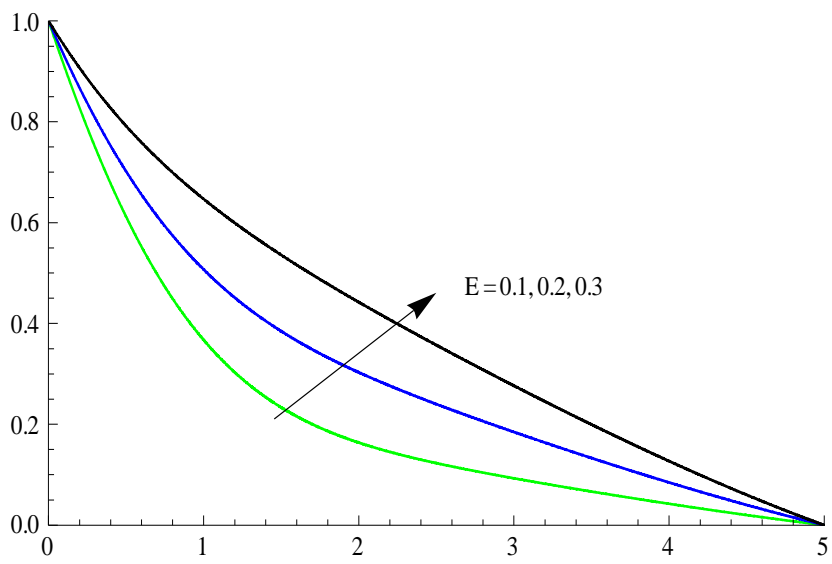

(b)

Fig.7. Temperature profile $\theta(\eta)$ for different values of $E$ by taking (a) $\beta=0.1$ and (b) $\beta=2$ for fixed value of

$$
M=1, N=1, R=1 \text { and } P_{r}=1
$$

The numerical value of skin friction co-efficient and Nusselt number are computed using the Mathematica software and are displayed in the tabular form in Tables 2 to 9 for various value of $\beta, N, M, P_{r}, R$ and $E$. Table 2. shows that with increase of $\beta$, numerical values of wall shear stress decreases provided that $\beta<1$, but increases provided that $\beta>1$ respectively. This is because of the nature of the value of $f^{\prime \prime}(0)$ which is positive when $\beta>1$ and negative when $\beta<1$.

Table 3 and Table 4 give the computed value of the dimensionless shear stress for various value of $M$ for the cases $\beta<1$ and $\beta>1$ respectively. It may observe that for a fixed value of $\beta$ the wall shear stress decreases with increase in the value of $M$ for the case $\beta<1$, but increases for the case $\beta>1$.

Table 5 gives the computed value of the dimensionless shear stress for various value of $N$ for the cases $\beta<1$ and $\beta>1$ respectively. It may observe that for a fixed value of $\beta$ the wall shear stress decreases with increase in the value of $N$ provided that $\beta<1$, but increases provided that $\beta>1$.

Table 6 display a decrease of thermal boundary layer thickness with increasing value of Prandtl number for the cases $\beta<1$ and $\beta>1$ respectively. In heat transfer problems, the Prandtl number $P_{r}$ controls the relative thickening of momentum and the thermal boundary layers. Fluid with lower Prandtl number wills posses higher thermal conductivities so that heat can diffuse from the sheet faster than for higher $P_{r}$ fluids. Hence Prandtl number can be used to increase the rate of cooling.

Table 7 displays a decrease of Nusselt number with increasing value of Radiation parameter for the cases $\beta<1$ and $\beta>1$ respectively because Radiation/absorption has tendency to reduce rate of heat transfer. Therefore, Nusselt number (rate of 
heat transfer) decreases with increase in radiation parameter. Table 8 and Table 9 display an increase in Nusselt number with increasing value of Eckert number because Heat transfer increases with increase in viscous dissipation which is measured by Eckert number. So, the Nusselt number increases with increase in Eckert number for the cases $\beta<1$ and $\beta>1$ respectively

Table. 1. Comparison of $\theta^{\prime}(0)$ for $\operatorname{Pr}=1$ when $M=0, N=0, R=0$ and $E=0$

\begin{tabular}{|c|c|c|}
\hline \multirow{2}{*}{$\beta$} & \multicolumn{2}{|c|}{$\theta^{\prime}(0)$} \\
\cline { 2 - 3 } & Mahapatrta et al (2002) & Present paper \\
\hline 0.1 & -0.603 & -0.603142 \\
\hline 0.2 & -0.625 & -0.625147 \\
\hline 2 & -0.974 & -0.973912 \\
\hline 3 & -1.124 & -1.134276 \\
\hline
\end{tabular}

Table. 2. Value of $-f^{\prime \prime}(0)$ for various value of $\beta, M, N, \operatorname{Pr}, R$, and $E$.

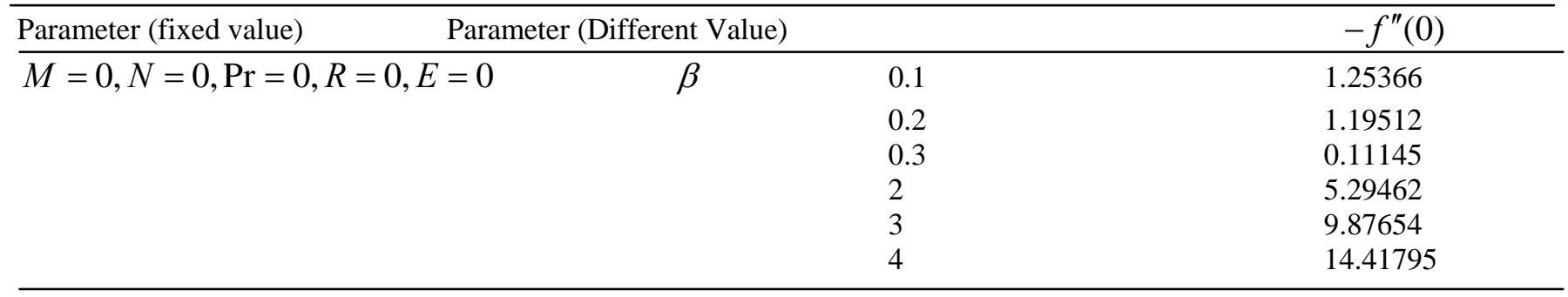

Table.3. Value of $-f^{\prime \prime}(0)$ for various value of $M$ for fixed $\beta=0.1, N=1, \operatorname{Pr}=1, R=1, E=0.1$ when $\beta>1$.

\begin{tabular}{|c|c|c|}
\hline Parameter (fixed value) & Parameter (Different Value) & $-f^{\prime \prime}(0)$ \\
\hline$N=1, P_{r}=1, R=1, E=0.1$ & $M$ & 1.79345 \\
\hline & $\begin{array}{l}2 \\
3\end{array}$ & $\begin{array}{l}3.34267 \\
4.20286\end{array}$ \\
\hline
\end{tabular}

Table.4. Value of $-f^{\prime \prime}(0)$ for various value of $M$ for fixed value of $\beta=2, N=1, \operatorname{Pr}=1, R=1, E=0.1$ when $\beta<1$.

\begin{tabular}{lccc}
\hline Parameter (fixed value) & Parameter (Different Value) & $-f^{\prime \prime}(0)$ \\
\hline$N=1, P_{r}=1, R=1, E=0.1$ & $M$ & 0.5 & 4.39678 \\
& & 1.0 & 4.43828 \\
& & 1.5 & 4.69732 \\
\hline
\end{tabular}

Table. 5.Value of $-f^{\prime \prime}(0)$ for various value of $N$ for fixed value of $M=1, \operatorname{Pr}=1, R=1, E=0.1$ for both cases.

\begin{tabular}{|c|c|c|c|}
\hline \multirow[t]{2}{*}{ Parameter (fixed value) } & \multirow[t]{2}{*}{ Parameter (Different Value) } & \multicolumn{2}{|c|}{$-f^{\prime \prime}(0)$} \\
\hline & & $\beta<1$ & $\beta>1$ \\
\hline \multirow[t]{2}{*}{$N=1, P_{r}=1, R=1, E=0.1$} & $N$ & 1.76718 & 4.53688 \\
\hline & $\begin{array}{l}1 \\
2\end{array}$ & $\begin{array}{l}2.54609 \\
4.58755\end{array}$ & $\begin{array}{l}4.65972 \\
4.86378\end{array}$ \\
\hline
\end{tabular}


Table. 6. Value of $-\theta^{\prime}(0)$ for various value of $\operatorname{Pr}$ for fixed value of $M=1, N=1, R=1, E=0.1$ for both cases.

\begin{tabular}{|c|c|c|c|}
\hline \multirow[t]{2}{*}{ Parameter (fixed value) } & \multirow[t]{2}{*}{ Parameter (Different Value) } & \multicolumn{2}{|c|}{$-\theta^{\prime}(0)$} \\
\hline & & $\beta<1$ & $\beta>1$ \\
\hline \multirow[t]{2}{*}{$N=1, P_{r}=1, R=1, E=0.1$} & $\operatorname{Pr}$ & 0.75903 & 0.97818 \\
\hline & $\begin{array}{l}2 \\
3\end{array}$ & $\begin{array}{l}0.84548 \\
1.71293\end{array}$ & $\begin{array}{l}1.37410 \\
1.703891\end{array}$ \\
\hline
\end{tabular}

Table. 7.Value of $-\theta^{\prime}(0)$ for various value of $R$ for fixed value of $M=1, N=1, \operatorname{Pr}=1, E=0.1$ for both cases.

\begin{tabular}{|c|c|c|c|}
\hline \multirow[t]{2}{*}{ Parameter (fixed value) } & \multirow[t]{2}{*}{ Parameter (Different Value) } & \multicolumn{2}{|c|}{$-\theta^{\prime}(0)$} \\
\hline & & $\beta<1$ & $\beta>1$ \\
\hline \multirow[t]{2}{*}{$N=1, P_{r}=1, R=1, E=0.1$} & $R$ & 0.75903 & 0.97876 \\
\hline & $\begin{array}{l}2 \\
3\end{array}$ & $\begin{array}{l}0.84361 \\
0.98323\end{array}$ & $\begin{array}{l}1.24063 \\
1.34015\end{array}$ \\
\hline
\end{tabular}

Table. 8. Value of $-\theta^{\prime}(0)$ for various value of $E$ for fixed value of $M=2, N=1, \operatorname{Pr}=1, R=1$ for the case $\beta<1$.

\begin{tabular}{llll}
\hline Parameter (fixed value) & Parameter (Different Value) & \\
\hline$N=1, P_{r}=1, R=1, E=0.1$ & \multirow{2}{*}{$E$} & 0.1 & 0.75903 \\
& & 0.9 & 0.47281 \\
& & 0.15 & 0.39754
\end{tabular}

Table. 9.Value of $-\theta^{\prime}(0)$ for various value of $E$ for fixed value of $M=2, N=1, \operatorname{Pr}=1, R=1$ for the case $\beta>1$.

\begin{tabular}{|c|c|c|}
\hline Parameter (fixed value) & Parameter (Different Value) & $-\theta^{\prime}(0)$ \\
\hline$N=1, P_{r}=1, R=1, E=0.1$ & $E$ & 0.98651 \\
\hline & $\begin{array}{l}2 \\
3\end{array}$ & $\begin{array}{l}0.83803 \\
0.67685\end{array}$ \\
\hline
\end{tabular}

\section{Conclusions}

The effect of thermal radiation and heat transfer over an unsteady two dimensional viscous, incompressible fluid flow of an electrically conducting fluid on exponentially stretching surface embedded in a porous medium has been investigated. Fluid is considered in the influence of transverse magnetic field and thermal radiation. Numerical solution for governing equation has been obtained. Which allow the computation of the flow and heat transfer characteristic for various values of the magnetic parameter, permeability parameter, radiation parameter, Eckert number and Prandtl number.

The main result of the paper can be summarized as follows:

- The boundary layer thickness decreases as $\beta$ increases. The increase in the value of $\beta$ implies that free stream velocity increases in comparison to stretching velocity, which results in the increase in pressure and straining motion near stagnation point.

- When Hartman number increases the velocity profiles decreases when $\beta<1$. In case when $\beta>1$, which is just opposite to $\beta<1$, the velocity profiles increase with the increase in the Hartmann number. The shear stress at the sheet decreases 
due to increase in the Hartmann number when $\beta<1$, while it increases with the increase in the Hartmann number when $\beta>1$ which explains the results presented in tab. 4 .

- The effect of porosity parameter $N$ depends on $\beta$. When $\beta<1$, velocity profile decreases as porosity parameter increases. The velocity profiles increase with the increase in the porosity parameter when $\beta>1$.

- The temperature profile decreases with an increase in the Prandtl number. This is in agreement with the physical fact that at higher Prandtl number, fluid has a thinner thermal boundary layer .

- With increase in radiation parameter the temperature profiles decrease but with increase in Eckert number the temperature profiles increases.

\section{Nomenclature}

$\begin{array}{cl}\beta & \text { Velocity ratio parameter } \\ \rho & \text { Fluid density } \\ v & \text { Kinematic viscosity } \\ \mu & \text { Dynamic viscosity } \\ T & \text { Temperature } \\ k & \text { Thermal conductivity } \\ c_{p} & \text { Specific heat and } \\ q_{r} & \text { Radiative heat flux. } \\ T_{0} \text { and } T_{\infty} & \text { Temperatures at and far away from the plate } \\ l & \text { Constant. } \\ \sigma . & \text { Mean absorption co-efficient } \\ \sigma & \text { Stefen Boltzman constant } \\ \text { a } & \text { Stretching sheet parameter } \\ \mathrm{b} & \text { free stream velocity parameter } \\ M & \text { Magnetic parameter. } \\ N & \text { Permeability parameter } \\ R & \text { Radiation parameter } \\ P_{r} & \text { Prandtl number } \\ E & \text { Eckert number. } \\ \psi & \text { Stream function } \\ \eta & \text { Similarity variable }\end{array}$

\section{References}

Al-Harbi, S. M., 2007, Numerical Study of Heat Transfer over Permeable Stretching Surface with Variable Viscosity and Thermal Diffusivity in Uniform Magnetic Field, Soochow Journal of Mathematics, Vol. 3, No. 2, pp. 229-240.

Anderson, H.I. 1992, MHD flow of a viscoelastic fluid past a stretching surface, Acta Mech., Vol. 95, pp. 227-230.

Ariel,P.D., Hayat,T.and Asghar., S. 2006, The flow of an elastico-viscous fluid past a stretching sheet with partial slip, Acta Mech., Vol. 187, pp 29-35.

Attia, H. A., 2003, Hydromagnetic Stagnation Point Flow with Heat Transfer over a Permeable Surface, The Arabian Journal for Science and Engineering, Vol.18, pp. 107-112

Attia, H. A., 2000, Axisymmetric Stagnation Point Flow Towards a Stretching Surface in the Presence of a Uniform Magnetic Field with Heat Generation, Tamkang Journal of Science and Engineering ., Vol.10, No. 1, pp 11-16.

Bansal, J. L., 1977, Viscous Fluid Dynamics, Oxford \& IBH Pub. Co., New Delhi, India.

Bejan, A., Nield, D. A., 2006, Convection in Porous Media, 3rd ed., Springer, New York, USA.

Bhattacharyya, K. and Mukhopdhay, S., 2011, Slip effect of unsteady boundary layer stagnation point flow and heat transfer towards a stretching sheet, Chinese physics letter, Vol. 28, No. 9, 94702(1-4).

Bhattacharyya, k., 201,. Effects of radiation and heat source/sink on unsteady MHD boundary layer flow and heat transfer over a shrinking sheet with suction/injection, Front. Chem. Sci. Eng. Vol. 5, pp. 376-384.

Chen C.K., Char M.I., Heat transfer of a continuous stretching surface with suction or blowing, J. Math. Anal. Appl. Vol.135, pp. 568-580. 
Chen, C. H, .2008, MHD Mixed convection of a power law fluid past a stretching surface in the presence of thermal radiation and internal heat generation /absorption, Int. J. of Nonlinear Mechanics, Vol. 44, No. 6, pp. 296-603.

Chiam, T.C., 1994, Stagnation-point flow towards a stretching plate, J.Phys. Soc. Jpn. Vol. 63, pp. 2443-2444.

Cortell, R., 2006, Flow and heat transfer of an electrically conducting fluid of second grade over a stretching sheet subject to suction and to a transverse magnetic field, Int. J. Heat Mass Transfer., Vol 49, pp. 1851-1856.

Cortell, R., 2007, Viscous flow and heat transfer over a nonlinearly stretching sheet, Appl. Math. Comput. Vol.184, pp. 864-873.

Elbashbeshy, E.M.A., 2001, Heat transfer over an exponentially stretching continuous surface with suction, Arch. Mech., Vol. 53, No. 6, pp. 643-651.

Eldabe Nabil, T.M. and Mohamed Mona, A.A,. 2002, Heat and mass transfer in hydromagnetic flow of the non-Newtonian fluid with heat source over an accelerating surface through a porous medium medium, Chaos, Solutions and Fractals, ,Vol.13, pp. 907-917.

Ghaly, A. Y., Elbarbary, E. M. E., 2002, Radiation Effect on MHD Free-Convection Flow of a Gas at a Stretching Surface with a Uniform Free Stream, Journal of Applied Mathematics, Vol. 2, No. 2, pp. 93-103.

Gupta. P. S.and Gupta, A. S., 1977, Heat and mass transfer on a stretching sheet with suction and blowing, Can. J. Chem. Eng. Vol. 55, pp. 744-746.

Grubka, L.J. and Bobba, K.M., 1985, Heat transfer characteristics of a continuous stretching surface with variable temperature, ASME J. Heat Transfer. Vol .07, pp. 248-250.

Hayat,T. and Sajid, M.2007, Analytic solution for axisymmetric flow and heat transfer of a second grade fluid past a stretching sheet, Int. J. Heat Mass Transfer., Vol. 50 ,pp 75-84.

Hiemenz,k., 1911, The boundary layer on a same shape ingen in the Flussigkeits current logged-dived straight circular cylinder, Dingler's Poly. J. Vol. 326, pp. 321-332

Ishak, A., Pop, I., Nazar, R, .2007, Steady and unsteady boundary layers due to a stretching vertical sheet in a porous medium using Darcy-Brinkman equation model, Int J.of Applied Mechanics and Engineering., Vol.3, pp. 623-637.

Ishak, A., 2011, MHD boundary layer flow due to an exponentially stretching sheet with radiation effect, Sains Malaysiana.,Vol. 40, pp. 391-395.

Jat, R. N., Chaudhary, S., 2009, MHD flow and heat transfer over a stretching sheet, Applied Mathematical Sciences, Vol. 26, pp. 1285-1294.

Khan, S. K., Sanjayanand, S., 2005, Viscoelastic boundary layer flow and heat transfer over an exponential stretching sheet, International Journal of Heat and Mass Transfer, Vol. 48. pp. 229-233.

Khan, S.K., 2006, Heat transfer in a viscoelastic fluid flow over a stretching surface with heat source/sink, suction/blowing and radiation, Int. J. Heat and Mass Transfer ., Vol.49, pp. 628-639.

Khan, S.K. and Sanjayanand, E., 2005. Viscoelastic boundary layer flow and heat transfer over an exponentially stretching sheet, Int. J. Heat Mass Transfer., Vol. 48, pp. 1534-1542.

Kumaran, V. and Ramanaiah, G., 1996,. A note on the flow over a stretching sheet, Acta Mech.,Vol 116, pp. 229-233.

Liao, S.J,. 2003, On the analytic solution of magnetohydrodynamic flows of non-Newtonian fluid over a stretching sheet, J. Fluid Mech., Vol. 488, pp 189-212.

Magyari, E and Keller, B., 1999. Heat and mass transfer in the boundary layers on an exponentially stretching continuous surface, J.Phys. D Appl. Phys., Vol.32, pp. 577-585.

Mahapatra, T.R., Gupta A.S., 2002, Heat transfer in stagnation-point flow towards a stretching sheet. Heat and MassTransfer., Vol. 38, pp. 517-521.

Mahapatra,T.R., Gupta,A. S.,2001, Magnetohydrodynamic stagnation-point flow towards a stretching sheet, Acta Mech., Vol.152, pp. 191-196.

Partha,M.K., Rajasekhar, Murthy,G.P., 2005, Effect of viscous dissipation on the mixed convection heat transfer from an exponentially stretching surface, Heat Mass Transfer., Vol. 41, pp. 360-366.

Pavlov K.B., 1974, Magnetohydrodynamic flow of an incompressible viscous fluid caused by the deformation of a plane surface, Magn. Gidrod. Vol.10, pp.146-148.

Pop, I., Pop, S. R., Grosan, T.,2004, Radiation effects on the flow near the stagnation point, Technische Mechanik, Vol. 25, No. 2, pp. 100-106.

Rashad, A. M. 2007, Radiative effect on heat transfer from a stretching surface in a porous medium, Int. J. of Appl. Math and Mech., Vol. 3, No. 4, pp. 14-23.

Sarpakaya., T.,1961, Flow of non-Newtonian fluids in a magnetic field, AIChE J., Vol.7, pp. 324-328.

Sajid, M. and Hayat, T., 2008. Influence of thermal radiation on the boundary layer flow due to an exponentially stretching sheet, Int. Comm. Heat Mass Transfer., Vol.35, pp. 347-356.

Sakiadis, B.C., 1961, Boundary layer behaviour on continuous solid surface: II - Boundary layer on a continuous flat surface AIChE.J., Vol.7, pp. 221-225.

Siddheshwa,,P.G. and Mahabaleshwar, U.S., 2005, Effect of radiation and heat source on MHD flow of a viscoelastic liquid and heat transfer over a stretching sheet, Int. J. Non-Linear Mech.,Vol. 40, pp. 807-820.

Schlichting, H., 1979, Boundary Layer Theory, 7th ed., McGraw-Hill, New York, USA. 
Singh, G., Sharma, P. R., 200,. Effects of Ohmic heating and viscous dissipation on steady MHD flow near a stagnation point on an isothermal stretching sheet, Thermal Science, Vol. 3, No. 1, pp. 5-12.

Timol, M. G., Patel, M. 2009, Numerical solution of steady two-dimensional MHD forward stagnation point flow, Applied Mathematical Sciences, Vol. 3, No. 4, pp. 187-193.

Vajravelu K.., Viscous flow over a nonlinearly stretching sheet, Appl. Math. Comput. Vol. 124, pp. 281-288.

Vajravelu, K.., 1994, Flow and Heat Transfer in a saturated porous medium. ZAMM ., Vol.74, No. 12, pp. 605-614.

Venkateswalu, S. Suryanarayana Rao., K.V.and Rambupal, B.R., 2011, Finite difference analysis on convective heat transfer flow through a porous medium in a vertical channel with magnetic field. Int. J. of Appl. Math and Mech., vol. 7 (7), pp. 74-94.

White, F. M,. 2006, Viscous Fluid Flow, 3rd ed., McGraw-Hill, Singapore.

Zheng L., Wang L., Zhang X., 2011, Analytic solutions of unsteady boundary flow and heat transfer on a permeable stretching sheet with non-uniform heat source/sink, Commun. Nonlinear Sci. Numer. Simulat. Vol.16, pp.731-740.

\section{Biographical notes}

Prof. (Mrs.) Bani Mukherjee (nee Chatterjee), M.Sc, D.I.I.T (IIT, KGP) and PhD in Mathematics (IIT, KGP) is a faculty in the Department of Applied Mathematics, Indian School of Mines, Dhanbad since 21st Dec, 1984. A number of students of M.Sc. (Math \& Comp.) and M.Phil (Maths) were done dissertation work under her guidance. Prof. Mukherjee has served as reviewers in many International/National journals. She has published several independent and joint research papers in international \& national journals. Many research papers are under preparation, which are joint and independent in nature. Her specialization and research interest is as follow: Relativistic Fluid Mechanics, Operations Research, Mathematical Modeling and Newtonian and Non-Newtonian Fluid Dynamics.

Narayan Prasad received M. Sc. Degree in Mathematics from Vinova Bhave University, Hazaribag, Jharkhand, India in 2008. In the year 2010, he has joined as a full time research scholar in the Department of Applied Mathematics to do the research work leading to Ph. D. degree on the research topic entitled "Mathematical Modelling of Some Boundary value Problem in Hydrodynamics ”. He has 2 years of research experience.

Received February 2013

Accepted November 2013

Final acceptance in revised form November 2013 